\title{
The optimization of DG capacity using PSO based on immune
}

\author{
algorithm \\ Rui Ma \\ North China Electric Power University Baoding, Baoding 071000, China \\ 1150229173@qq.com
}

Keywords: particle swarm optimization (PSO) algorithm, active distributed generation (ADN), immune algorithm, active management (AM)

Abstract: For the model of optimization of DG accession capacity, improved Newton-Raphson algorithm is used to calculate the power flow. The particle swarm optimization algorithm based on immune is used to optimize the model, and the maximum capacity of DG is obtained. Finally, the IEEE 33-bus distribution system [1] is used as an example to verify, the power flow and node voltage are calculated separately before and after the DG connected to distribution network and the system indicators are compared to come into four conclusions.

\section{1、Introduction}

The DG maximum accession capacity model is put forward firstly. Then Particle swarm optimization (PSO) based on immune algorithm is used to solve the optimization problem. Finally, IEEE 33-bus distribution system is used to verify four conclusions. A useful exploration has been done in the area of larger capacity and higher percentage DG connected to the ADN [2].

\section{2、The model of DG maximum accession capacity}

The objective function this paper used to describe the maximum admission capacity of DG is:

$$
\max \sum_{i=1}^{n} P_{D G i}
$$

In the case of safe and stable operation of the network, the constraint includes the power flow constraint, the node voltage constraint and the line power constraint, as shown in equations (2) to (7):

Power flow constraint:

$$
\begin{aligned}
& P_{G i}-P_{L i}=U_{i} \sum_{k \in A(i)} U_{k}\left(G_{I K} \cos \delta_{i k}+B_{i k} \sin \delta_{i k}\right) \\
& Q_{G i}-Q_{L i}=U_{i} \sum_{k \in A(i)} U_{k}\left(G_{I K} \sin \delta_{i k}-B_{i k} \cos \delta_{i k}\right)
\end{aligned}
$$

Node voltage constraint:

$$
V_{\text {imin }} \leq V_{i} \leq V_{\text {imax }}
$$

Branch power constraint:

$$
S_{j} \leq S_{\text {jmax }}
$$

DG power constraint: 


$$
P_{D G i m i n} \leq P_{D G i} \leq P_{D G i m a x}
$$

OLTC tapping constraint:

$$
T_{k \min } \leq T_{k} \leq T_{k \max }
$$

Reactive Power Compensation Device constraint:

$$
Q_{\text {Cimin }} \leq Q_{C i} \leq Q_{\text {Cimax }}
$$

\section{3、 The solution to the problem}

\subsection{PSO based on immune algorithm}

Particle swarm optimization is a group intelligent algorithm, which is a kind of fireworks calculation based on group intelligent method. Its advantages are good in practical effect, high precision, simple and intuitive, and easy to implement and versatile, especially for engineering optimization.

The advantages and disadvantages between various PSO depend on their global search ability, local search ability and convergence speed. PSO based on Immune is combined PSO with immune algorithm. On one hand, it improves its global search ability and on the other hand it accelerates the convergence speed.

\subsection{The steps of optimization of DG accession capacity}

The calculation steps of the optimization of DG using PSO based on immune algorithm are as follows:

(1) Enter the network initial parameters, including the learning factors $c_{1}$ and $c_{2}$, the max iterations $K_{\max }$, the max inertia weight $w_{\max }$, the min inertia weight $w_{\min }$, the precision $\varepsilon$, the population size $N$;

(2) Calculate the network power flow, solve the objective function, record the value of max objective function $F_{\text {best }}$ (which is the max total power), the value of each particle $P_{b e s t}$ (which is the power of each DG).

(3) Update the number of the iterations. And the inertia weight is calculated according to the formula: $w=w_{\max }-k\left(w_{\max }-w_{\min }\right) / k_{\max }$.

(4) Calculate the new power flow and the objective function value of each particle. Update and record the largest objective function value and particle position;

(5) Determine whether the maximum iterations are up to the maximum. If it reaches, terminate the program; otherwise repeat steps (3), (4);

(6) Output DG total accession capacity and each DG power capacity;

Optimization of the configuration process is shown in Figure 1

\section{4、 Example analysis}

In this paper, IEEE 33-bus distribution system used as the test system. Structure is shown in Figure 3. The system has one power supply, five contact switch branches, 32 branches and 33 nodes. The base voltage is $12.66 \mathrm{kV}$, and the base apparent power is 10MVA. In the example analysis, DG is connected to three nodes 2,9,17. Use PSO 
based on immune algorithm to solve the optimization problem. In the paper, DG is viewed as PQ node, which power factor is 0.9.

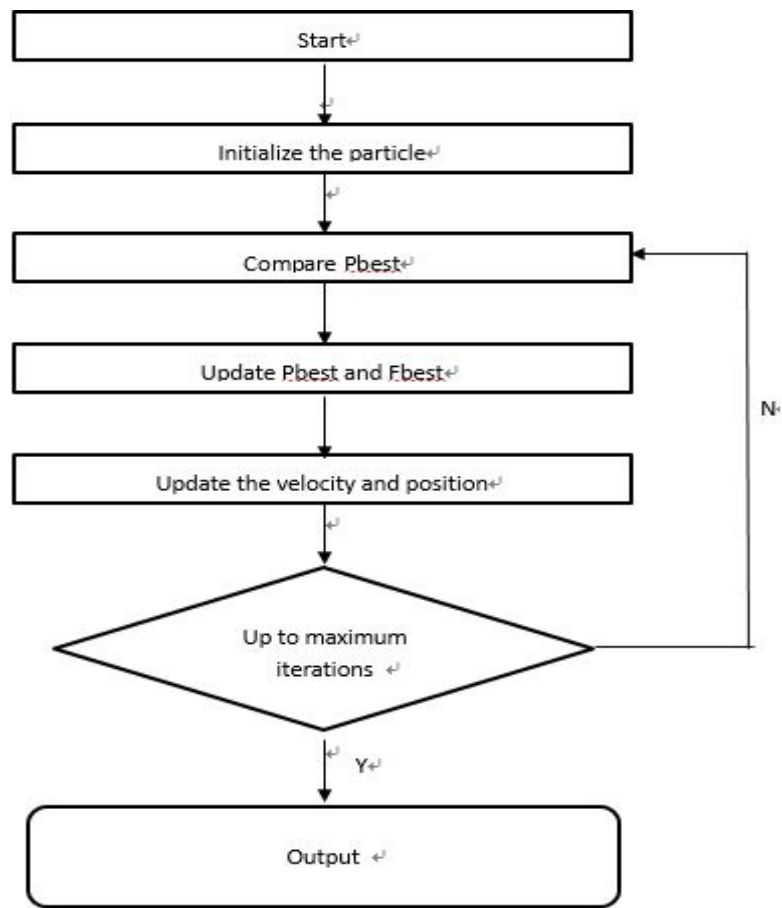

Figure 1 The steps of optimization

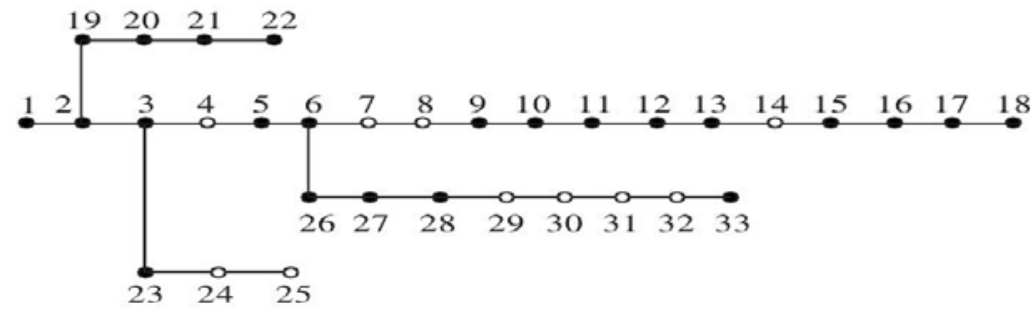

Figure 2 IEEE 33-bus distribution system

Table 1 shows the calculation results of the average of node voltage amplitude, the minimum voltage amplitude, the maximum voltage amplitude, the active power loss and the voltage level index before DG is connected to the network.

Table 1 System indicators without DG

\begin{tabular}{|c|c|c|c|}
\hline The average voltage & The min voltage & The max voltage & power loss \\
\hline 0.9485 & 0.913 & 1 & 0.203 \\
\hline
\end{tabular}

In the case of DG accession capacity is unknown; PSO based on immune algorithm is used to solve the model. The result is that the total of DG accession capacity is $1.14 \mathrm{MW}$. The specific optimized configuration results are shown in Table 2. Based on the optimization program, the average of node voltage amplitude, the minimum node voltage amplitude, the maximum node voltage amplitude, the active power loss and the voltage level index with DG connected to the network are calculated. The results are shown in Table 3. 
Table 2 The admission capacity of DG connection

\begin{tabular}{|c|c|c|c|}
\hline Node & 2 & 9 & 17 \\
\hline Capacity & 0.4 & 0.35 & 0.22 \\
\hline
\end{tabular}

According to the optimization program shown in Table 2, the active power loss of the distribution system reduces from $0.205 \mathrm{MW}$ to $0.105 \mathrm{MW}$ which reduced by $48.28 \%$. The average of node voltage amplitude increases from 0.9485 to 0.9622 , the minimum node voltage increases from 0.913 to 0.930 , which meets the standard requirements. At this situation, the total accession capacity of the DG connected to distribution system account network total load for $26.5 \%$.

Table 3 System indicators with DG

\begin{tabular}{|c|c|c|c|}
\hline The average voltage & The min voltage & The max voltage & power loss \\
\hline 0.9622 & 0.930 & 1 & 0.105 \\
\hline
\end{tabular}

It can be seen that the DG optimization program not only ensures the safe and reliable operation of the distribution network under the high proportion of DG, but also reduces the active loss of the distribution network and improves the node voltage level and the network voltage distribution.

Meanwhile the admission capacity between ADN and the traditional distribution network have been compared.

In the view of the AM, Table 4 lists the maximum accession capacity of nodes connected to the nodes, respectively 2,9,17 of the front, middle and last ends of the distribution system. It can be seen that the DG accession capacity considering AM is larger than it without active management model, because the active management method can control the DG output, adjust the OLTC tap and control the reactive power compensation to make the network operating under the security range.

Table 1 Active and no-active management

\begin{tabular}{|c|c|c|c|}
\hline Node & 2 & 9 & 17 \\
\hline Active & 0.4 & 0.35 & 0.22 \\
\hline No-active & 0.39 & 0.30 & 0.16 \\
\hline
\end{tabular}

\section{4、Summary}

The model of maximum total DG power in ADN is established and a new corresponding algorithm is put forward. Meanwhile, the IEEE33 node network is used to verify the following conclusions:

(1) The admission capacity of DG account the total load for 31.5\%, which exceeds the traditional capacity of DG planning.

(2) Active management method can improve the DG accession capacity. The capacity of corresponding node 2,9,17 respectively is lager 3\%, $15 \%, 42 \%$ than traditional distribution network. The capacity of the node which is closer to the end is larger than the front, which is about $44 \%$ of the end.

(3) PSO based on immune algorithms not only has higher precision, but also calculates quickly which is very appropriately applied in the electric power system.

\section{Reference}

[1] Dugan R C, McDermott T E. Distributed generation [J]. IEEE Industry Application Magazine, 2002, 8(2), 19-25.

[2] Martins V F, Borges C L T. Active distribution network integrated planning 
incorporating distributed generation and load response uncertainties [J]. IEEE Transactions on Power Systems, 2011, 26(4): 2164-2172. 